\title{
Evolving to an Ideal Synthesis of Molnupiravir, an Investigational Treatment for COVID-19
}

\author{
Tamas Benkovics, ${ }^{1, \#}$ John A. McIntosh, ${ }^{1, \#}$ Steven M. Silverman, ${ }^{1, \#}$ Jongrock Kong, ${ }^{1}$ Peter Maligres, ${ }^{1}$ \\ Tetsuji Itoh, ${ }^{1}$ Hao Yang, ${ }^{1}$ Mark A. Huffman, ${ }^{1}$ Deeptak Verma, ${ }^{1}$ Weilan Pan, ${ }^{1}$ Hsing-I Ho, ${ }^{1}$ Jonathan \\ Vroom, ${ }^{2}$ Anders Knight, ${ }^{2}$ Jessica Hurtak, ${ }^{2}$ William Morris, ${ }^{1}$ Neil A. Strotman, ${ }^{1}$ Grant Murphy, ${ }^{1}$ Kevin M. \\ Maloney, ${ }^{1}$ Patrick S. Fier ${ }^{1, *}$
}

${ }^{1}$ Department of Process Research and Development, Merck \& Co., Inc., Rahway, New Jersey 07065, United States

${ }^{2}$ Codexis, Inc., 200 Penobscot Drive, Redwood City, CA 94063, USA

\section{Supporting Information Placeholder}

\begin{abstract}
Molnupiravir (MK-4482) is an investigational direct-acting antiviral agent that is under development for the treatment of COVID-19. Given the potential high demand for this compound, it was critical to develop a sustainable and efficient synthesis from commodity raw materials. The three-step route that we report here embodies the shortest possible synthesis to molnupiravir, and was enabled through the invention of a novel biocatalytic cascade and final condensation step. Each step occurs in over $95 \%$ yield and only utilizes widely available commodity reagents and simple operations. Compared to the initial route, the new route is $70 \%$ shorter, and approximately seven-fold higher in overall yield.
\end{abstract}

The ongoing COVID-19 pandemic caused by the SARS-CoV-2 virus has been devastating to the global population, with well-over 1.5 million deaths and rising infection rates around the world. ${ }^{1}$ In addition to novel vaccines, the development and supply of a directacting antiviral (DAA) compound to treat patients with COVID-19 is critical to ending the pandemic. To that end, molnupiravir (MK4482, formerly EIDD-2801) is an orally-dosed DAA agent currently in clinical trials for the treatment of patients with mild, moderate, or severe COVID-19. ${ }^{2}$ Given the anticipated widespread demand, the primary challenge of this effort is ensuring a robust manufacturing route that could supply hundreds of metric tons of molnupiravir across the globe.

To date, reported syntheses of molnupiravir have relied on building blocks, reagents, and reaction conditions that do not meet our criteria for an aspirational synthesis (Figure 1A). ${ }^{3}$ It is essential to use only true commodity raw materials available on multi-metric ton scale, processes, reagents, in quantities that are inherently sustainable and green, and to establish a route that can quickly deliver high quality API without the need for chromatography. By this assessment, neither cytidine nor uridine should be considered as true commodity raw materials, as both are typically prepared in at least four steps from ribose, their syntheses are not inherently green, and each has supply chain risks on the scales needed. Thus, we focused exclusively on ambitious routes that use ribose as the starting material. In order to meet these aspirational targets, we invested in the development of a route that best aligned with the criteria listed above, and would allow facile isolation of intermediates and API. However, the identified route (Figure 1B, for additional routes investigated, see the Supporting Information) relies on multiple unknown transformations (vide infra), that would require enzyme evolution, reaction invention, and process development to ensure molnupiravir could be made in high-purity from only commodity reagents and raw materials. Herein we present the development of a high-yielding three-step route that incorporates a novel enzymatic cascade to prepare to molnupiravir from true commodity chemicals, enabling worldwide access to this critical therapeutic.

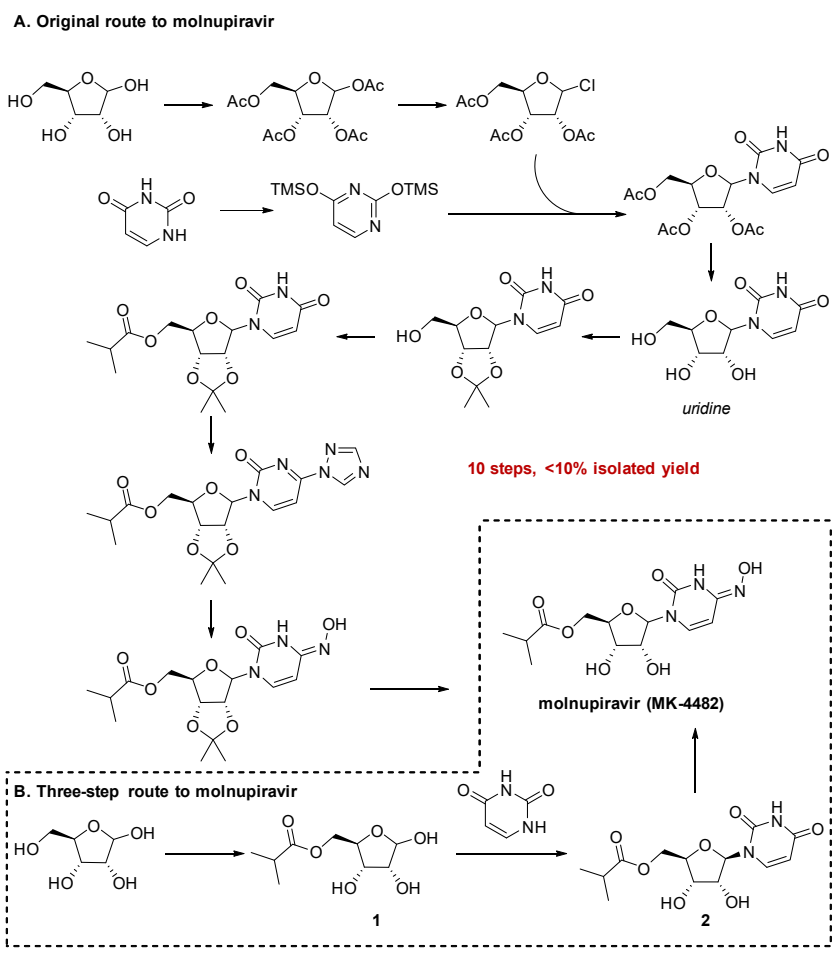

Figure 1. Original route and new three-step route chosen for the synthesis of molnupiravir.

Our synthesis begins with the selective esterification of ribose at the primary alcohol. While selective functionalization of a primary 
alcohol in the presence of secondary alcohols seemingly represents a simple transformation, this step is inherently difficult for several reasons: i) ribose adopts a pyranose form in many solvents ${ }^{4}$ in which the primary alcohol is present as a hemi-acetal, ii) ribose has poor solubility in inert solvents that are of relevance to process chemistry, iii) the ester product is more soluble than ribose leading to potential over-reaction at the other alcohol positions, and iv) the product is highly water soluble and poorly crystalline making separation from other components difficult. The reactivity challenges can be well-addressed by using biocatalysis, whereby the deployment of an enzyme would be expected to obviate the known selectivity challenges. Toward this goal, a panel of lipase enzymes $^{5}$ were investigated with $O$-isobutyryl acetone oxime ${ }^{6}$ as the isobutyryl donor, and proof of concept was rapidly obtained with multiple commercial lipases to afford $\mathbf{1}$ in modest yields (see Supporting Information). Consistent with our hypothesis, selectivity for the desired product was high, with only trace amounts of any side-products formed.

Given the demonstration of selective esterification using lipase enzymes, and the known challenge of product isolation, efforts were focused on the use of Novozym 435, an immobilized CalB lipase available in multi-metric ton quantities from Novozymes, to effect the esterification. ${ }^{7}$ Simultaneously, we shifted to the use of isobutyric anhydride as the isobutyryl donor, as it is a commodity raw material in-line with our aspirational goals, in contrast to the oxime reagent. Through a survey of solvents and enzyme loadings (Scheme 1), it was found that the esterification of ribose in acetone with $10 \mathrm{wt} \%$ Novozym 435 provided the desired 5-isobutyryl ribose in $94 \%$ yield (entry 8 ), with only a small amount of di-ester byproducts formed. As parallel development revealed that the following step in the synthesis should be carried out under aqueous conditions, and the ribose ester was poorly crystalline and highly water soluble, the decision was made to process $\mathbf{1}$ as a solution in water and feed the aqueous solution directly into the next step. After completion, the reaction mixture was filtered to remove the immobilized enzyme, the solvent was switched to MTBE, and the product was extracted efficiently into water, with the excess isobutyric anhydride and most of the isobutyric acid byproduct removed in the organic phase. The aqueous solution of the product 1 had high purity, was stable for over 1 month, and most importantly was suitable for use directly in the next step of the synthesis.

Scheme 1. Esterification of ribose catalyzed by Novozym $435^{a}$

$\begin{array}{ccccc}2 & \text { Novozym 435 } \\ \text { solvent } \\ 50^{\circ} \mathrm{C}, 24 \mathrm{~h}\end{array}$

${ }^{a}$ Conversions and assay yields based on ribose and measured using a calibrated UPLC-CAD instrument. Assay yields were not determined for entries with N/A in the yield column. Entry 8 afforded $92 \%$ isolated yield of 1 after workup.

The second step of the synthesis relies on a previously unknown reaction that incorporates uracil into the anomeric position of $\mathbf{1}$. Biocatalytic strategies to convert ribose derivatives to nucleosides typically require a free $\mathrm{C} 5$-hydroxyl to allow formation of ribose 5phophate. ${ }^{8}$ The nucleoside is then formed by enzyme-catalyzed isomerization to the corresponding $\alpha-1$-phosphate species followed by incorporation of the nucleobase with a nucleoside phosphorylase. However, this strategy can not be applied to $\mathbf{1}$, as it lacks a free 5-hydroxyl group, so a novel direct phosphorylation of the anomeric position was needed.

In Nature, direct 1-phosphorylation of 5-S-methylthioribose (MTR) occurs as part of the methionine salvage pathway and is catalyzed by MTR kinases. ${ }^{9}$ Although MTR kinases do not participate in nucleoside biosynthesis in Nature, and we are unaware of any previous biocatalytic applications, we hypothesized that an MTR kinase could be leveraged to generate a 5isobutyrylribose-1-phosphate intermediate (1-phosphate, Scheme 2 ), which we proposed could subsequently be converted by an appropriate nucleoside phosphorylase to 5 '-isobutyryluridine (2).

Scheme 2. Strategy to install uracil ring on 1 with MTR kinase and nucleoside phosphorylase enzymes

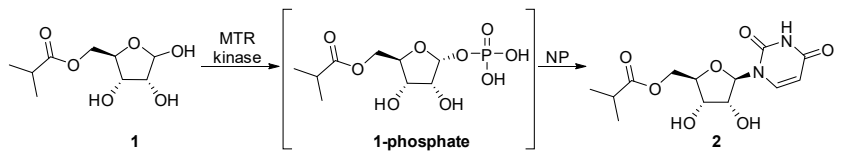

${ }^{a}$ For clarity, salt form of 1-phosphate is not shown

With this goal in mind, we initiated tandem screening efforts to identify an appropriate nucleoside phosphorylase and 1-kinase. Nucleoside phosphorylases ${ }^{10}$ catalyze equilibration between sugar 1-phosphate and the nucleoside, affording the opportunity to screen the desired reaction in the reverse direction starting from $\mathbf{2}$. We constructed a diverse panel of nucleoside phosphorylase enzymes and screened for the phosphorolysis of $\mathbf{2}$ to generate $\mathbf{1}$ and uracil. From this panel, we identified E. coli uridine phosphorylase (UP; URP-003 from Codexis) as the most active, providing nearly $40 \%$ cleavage in high-throughput screens.

With a suitable nucleoside phosphorylase in hand, we examined the 1-phosphorylation to convert 1 to $\mathbf{1}$-phosphate. ${ }^{8 \mathrm{c}}$ A panel of MTR kinase enzymes from diverse bacterial species was produced by heterologous expression in E. coli. The MTR kinases were then screened in a cascade reaction with $E$. coli uridine phosphorylase for the production of $\mathbf{2}$ from $\mathbf{1}$ and uracil in the presence of catalytic ATP and an ATP regeneration system consisting of acetate kinase (AcK) and propionyl phosphate $(\operatorname{PrP})^{11}$ as the stoichiometric phosphate donor. Through these efforts, we identified several MTR kinases that catalyzed the phosphorylation reaction in the enzymatic cascade to prepare $\mathbf{2}$, albeit with modest activity that would require significant improvement to enable a viable process to molnupiravir.

From these hits, the MTR kinase from Klebsiella spp. was chosen as a starting point for directed evolution. In phosphorylation reactions absent uridine phosphorylase, the 1-phosphate product could be observed by UPLC-CAD or UPLC-MS, which showed that the wild-type enzyme afforded 7:1 selectivity in favor of the desired $\alpha$-anomer. After only one round of engineering, the phosphorylation reaction could be run at high concentrations to produce 2 in $>99 \%$ conversion and $>99: 1$ diastereoselectivity. Additional rounds of evolution enabled significant reductions in enzyme loading. Likewise, E. coli UP was also subjected to engineering, and a $>3$-fold improvement in activity was realized after one round of evolution.

As the uracil incorporation reaction is reversible, removal of the inorganic phosphate byproduct was necessary to drive the reaction in the forward direction. This exclusion could be accomplished through the addition of sucrose and sucrose phosphorylase (SuP, Scheme 3) to sequester the phosphate as glucose 1-phosphate, in line with our previously reported synthesis of HIV therapeutic islatravir. ${ }^{8 a}$ While phosphate sequestration proved an effective 
strategy in increasing product formation, the use of a stoichiometric phosphate donor inherently leads to reaction inefficiencies, which we hypothesized could be addressed through the design of a phosphate recycling system. If successful, this phosphate recycling system would not only help to drive the equilibrium but also allow for elimination of sucrose and sucrose phosphorylase from the reaction mixture and reduction in loading of the phosphate donor, thereby achieving our ideal synthesis.

Scheme 3. Enzymatic cascade from 1 to 2 with a stoichiometric phosphate donor and inorganic phosphate removal system

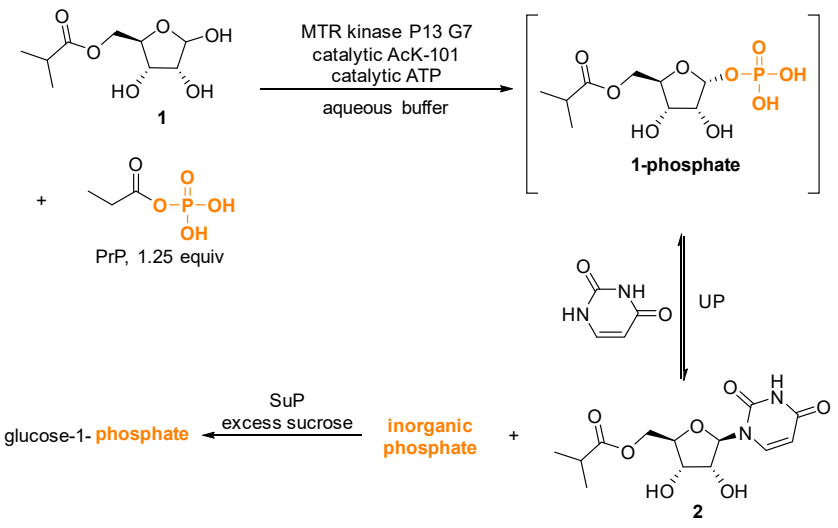

${ }^{a}$ For clarity, salt forms of phosphate species are not shown

In place of using a stoichiometric phosphate donor and inorganic phosphate scavenging system, an alternative approach was identified that utilizes the introduction of pyruvate oxidase (PO) to generate the high-energy phosphate donor in-situ (Scheme 4). PO catalyzes the oxygen-mediated formation of acetyl phosphate from pyruvic acid and inorganic phosphate. ${ }^{12}$ Since inorganic phosphate is the byproduct of the glycosylation reaction, we recognized that this strategy could enable transient formation and subsequent consumption of inorganic phosphate, avoiding both the need for a stoichiometric high-energy phosphate donor such as $\mathrm{PrP}$ and the need for separate phosphate sequestration. The use of PO for ATP regeneration has previously been reported to improve in-vitro protein synthesis, ${ }^{13}$ but we are unaware of other applications in biocatalysis. ${ }^{8,14}$ The full enzymatic cascade employs PO and AcK for ATP regeneration, ${ }^{14}$ MTR kinase for phosphorylation of $\mathbf{1}, \mathrm{UP}$ for uracil incorporation, and catalase to decompose hydrogen peroxide formed by PO. In total, this novel biocatalytic cascade can be run at a concentration of $>80 \mathrm{~g} / \mathrm{L}$ of 1 , forms the product in quantitative yield, and allows for facile isolation of $\mathbf{2}$ in $>99.5 \mathrm{wt} \%$ purity through extraction with 2-MeTHF and subsequent crystallization (see Supporting Information).

Scheme 4. Enzymatic cascade from 1 to 2 with PO and catalytic quantities of inorganic phosphate

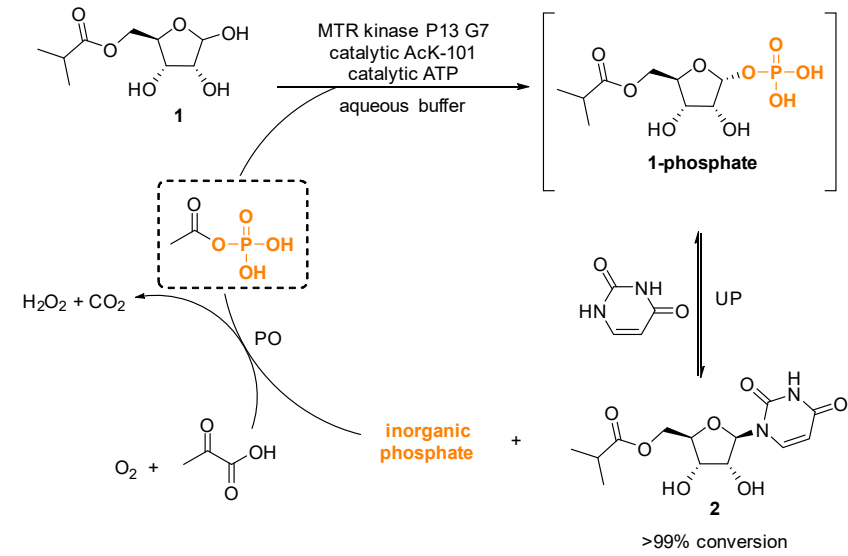

${ }^{a}$ For clarity, salt forms of phosphate species and catalase cycle are not shown. For additional details, see the Supporting Information.

Completion of the synthesis of molnupiravir requires conversion of the amidic-carbonyl in the uracil ring to the corresponding oxime. Initial investigation into this transformation found that phosphate activating agents, such as diethyl chlorophosphate, could be used to selectively activate the targeted carbonyl group, and subsequent reaction with hydroxylamine served to form the API. Despite the success of this approach, the use of chlorophosphate reagents was not pursued as these are classified as highly toxic and hazardous reagents, the use of which are not in line with our aspirational goals. We anticipated that simple trimethylsilyl reagents could promote the desired transformation through TMS-activation of the uracil ring, with the reaction ultimately driven by the formation of innocuous hexamethyldisiloxane $\left(\mathrm{TMS}_{2} \mathrm{O}\right) .{ }^{15}$ While $\mathrm{TMSCl}$ is inexpensive and readily available, it carries corrosivity risks and handling concerns, thus we focused solely on reactions that utilize hexamethyldisilazane (HMDS) as a mild dehydrating agent that is readily available on metric-ton scales.

Scheme 5. Conversion of 5'-isobutyryl uridine to molnupiravir

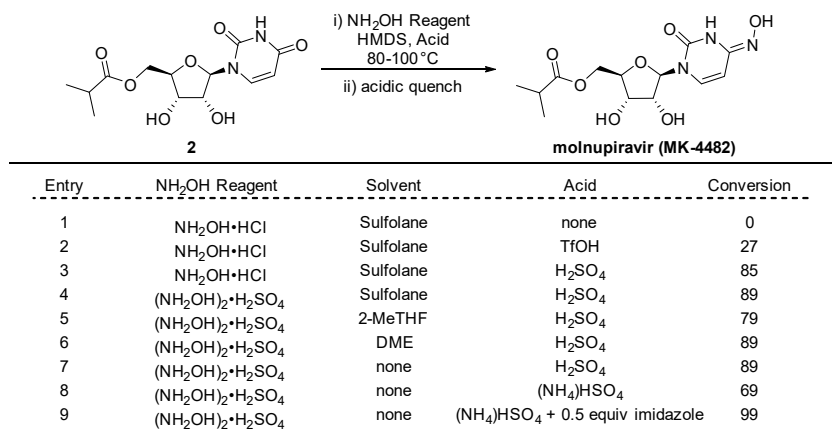

${ }^{a}$ Conversion after $24 \mathrm{~h}$ based on UPLC absorbance at $254 \mathrm{~nm}$. For additional details, see the Supporting Information.

Exploration of reaction conditions with HMDS, acidic promoters and various hydroxylamine reagents was conducted and provided proof of concept for API formation. Our initial hit utilized sulfolane as a co-solvent and triflic acid as the acidic promoter at $80{ }^{\circ} \mathrm{C}$ (Scheme 5, entry 2). Although sulfolane is a renewable solvent, it presents complications due to its high melting point for a solvent $\left(27.5^{\circ} \mathrm{C}\right)$ and the associated challenges with its removal in the API isolation. Similarly, the use of triflic acid led to fast reaction rates but was not pursued due to safety and handling concerns. Additionally, the harsh conditions required resulted in the formation of unidentified byproducts in the reaction mixture. 
Switching to sulfuric acid as the acid promoter provided the product in high conversion, and the use of the sulfate salt of hydroxylamine led to increased purity compared to previous reactions that had used the hydrochloride salt (Scheme 5, entries 34). Ultimately, it was found HMDS itself could be used as the reaction solvent (Scheme 5, entries 5-7), providing increased reaction rates relative to reactions in which it was used as a cosolvent, while providing operational simplicity in the reaction setup. The exotherm observed in mixing HMDS with sulfuric acid was circumvented through the use of bisulfate salts $\left(\mathrm{Na}, \mathrm{K}, \mathrm{NH}_{4}\right)$, with ammonium bisulfate providing the best results (Scheme 5, entry 8). Finally, the addition of a catalytic quantitiy of imidazole led to a dramatic increase on the reaction rate and conversion, likely through the in situ formation of TMS-imidazole as a silylation catalyst. ${ }^{16}$

After further development of the reaction conditions, conversion of 2 to molnupiravir in the presence of HMDS was shown to occur in nearly quantitative yield, without the generation of any significant side-products (Scheme 6). As anticipated, the initial product of the reaction is the bis-trimethylsilyl derivative of molnupiravir. This in-situ silylation allowed for facile removal of the inorganic reagents and byproducts via an aqueous wash without any loss of product. Following removal of the inorganic species, the TMS groups were easily cleaved by adjusting the $\mathrm{pH}$, after which the product could be crystallized, completing our novel three-step synthesis and providing molnupiravir in high yield and $>99.5 \mathrm{wt} \%$ purity.

Scheme 6. Conversion of 2 to bis-TMS molnupiravir, and isolation of molnupiravir ${ }^{a}$
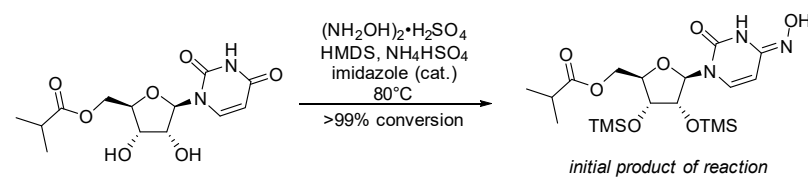

initial product of reaction

adjust $\mathrm{pH}$, wash with water to remove

inorganic compounds
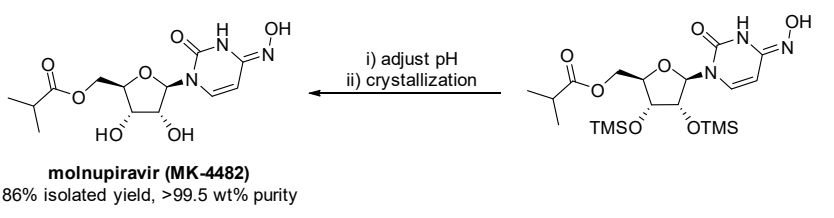

$86 \%$ isolated yield, $>99.5 \mathrm{wt} \%$ purity

${ }^{a}$ For additional details, see Supporting Information

Compared to the initial route ${ }^{3 \mathrm{a}, \mathrm{b}}$ and alternate syntheses ${ }^{3 \mathrm{c}, \mathrm{d}}$ reported for MK-4482, the three-step sequence reported here offers several notable improvements in yields and cycle times that was enabled through novel biocatalytic cascades and reaction development. Our three-step route (Scheme 7) embodies the shortest conceivable number of transformations from ribose, uracil, and an isobutyryl source. Furthermore, there is only a single isolated solid intermediate, which is isolated in high purity $(>99.5$ $\mathrm{wt} \%,<200 \mathrm{ppm}$ residual protein) through crystallization, and the API is step is high yielding and allows for pure $(>99.5 \mathrm{wt} \%)$ material to be isolated with simple operations. Finally, the route only utilizes simple process operations without the need for chromatography or large excesses of reagents or solvents, which are the hallmarks of a truly green and sustainable process.

Scheme 7. Summary of initial and new routes to molnupiravir from ribose and uracil
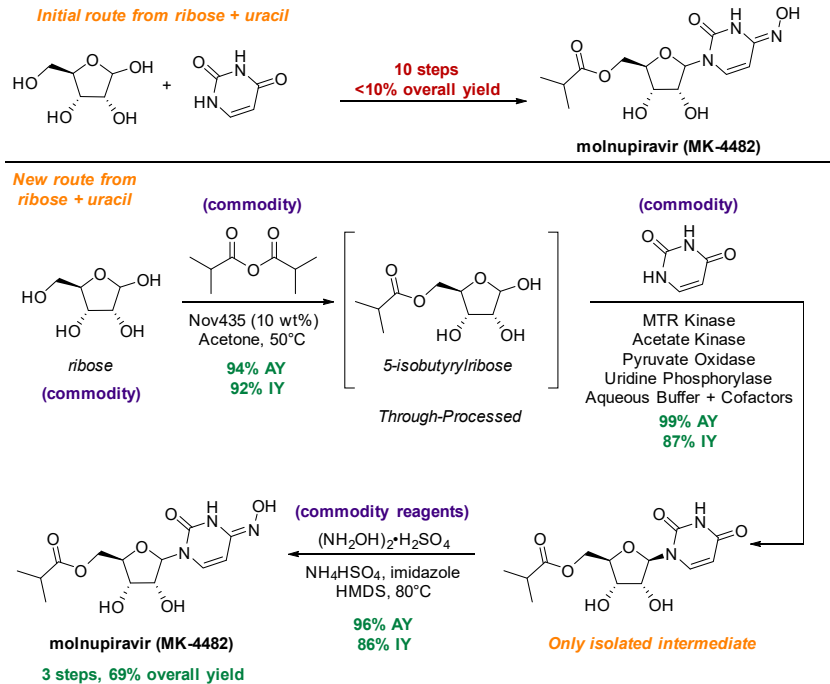

${ }^{a} \mathrm{AY}$ : Assay yield at the end of reaction, as determined using a calibrated UPLC instrument. IY: Isolated yields of $>99.5 \%$ purity material. For additional details, see the Supporting Information.

In summary, we have developed an efficient and inherently sustainable synthesis of molnupiravir that utilizes only true commodity raw materials, simple procedures, and robust reactions and isolations. The three-step route from ribose uses two enzymatic steps including a novel biocatalytic cascade that incorporates an innovative phosphate recycling system, has only a single isolated solid intermediate, and provides molnupiravir in high yield and purity. Compared to the initial route, the new route is $70 \%$ shorter, approximately 7-fold higher in overall yield, and enabled through the development and investment in novel chemistry. These synthetic discoveries provide the critical advancements to enable the availability and accessibility of the COVID-19 DAA molnupiravir to patients across the globe.

\section{AUTHOR INFORMATION}

\section{Corresponding Author}

*E-mail: patrick.fier@merck.com

\section{Author Contributions}

\#These authors contributed equally

\section{ACKNOWLEDGMENTS}

We thank our colleagues Rebecca Ruck, L.-C. Campeau, Guy Humphrey, and Kevin Campos for their support and helpful feedback. We thank Cecilia Bottecchia, Kevin Stone, Dan Lehnherr, Artis Klapars, Shane Grosser, Keith Mattern, and Gao Shang for helpful discussions. We thank Jiaxuan Yan, Joseph Gouker, Heather Wang, Jackson Hall, Rebecca Arvary, and Sherry Song for analytical support.

\section{REFERENCES}

(1) WHO Coronavirus Disease (COVID-19) Dashboard, https://covid19.who.int/ accessed December $14^{\text {th }}, 2020$

(2) (a) Merck and Ridgeback Bio Collaborate to Advance Development of Novel Antiviral Candidate, EIDD-2801. https://www.businesswire.com/news/home/20200526005229/en/ (b) Clinical trial study numbers MK-4482-001 and MK-4482-002.

(3) a) Painter, G. R.; Bluemling, G. R.; Natchus, M. G.; Guthrie, D. WO2019113462, 2018. (b) Painter, G. R.; Perryman, D.; Bluemling, G. R. WO2019173602, 2019. (c) Vasudevan, N.; Ahlqvist, G. P.; McGeough, C. P.; Paymode, D. J.; Cardoso, F. S. 
P.; Lucas, T.; Dietz, J.-P.; Opatz, T.; Jamison, T. F.; Gupton, F. B.; Snead, D. R. Chem. Commun. 2020, 56, 13363-13364. (d) Gopalsamuthiram, V.; Williams, C.; Noble, J.; Jamison, T. F.; Gupton, F. B.; Snead, D. R. SynLett 2020, 31, A-C.

(4) Drewa , K. N.; Zajiceka, J.; Bondob, G.; Bose, B.; Serianni, A. S. Carb. Res. 1998, 199-209.

(5) Chandra, P.; Enespa; Singh, R.; Arora, P. K. Microb. Cell Fact. 2020, 19, 169-210.

(6) Pulido, R.; Lopez Ortiz, F.; Gotor, V. J. Chem. Soc., Perkin Trans. 1. 1992, 21, 2891-2898.

(7) Ortiz, C.; Ferreira, M. L.; Barbosa, O.; dos Santos, J. C. S.; Rodrigues, R. C.; Berenguer-Murcia, Á.; Briand, L. E.; FernandezLafuente, R. Catal. Sci. Technol. 2019, 9, 2380-2420.

(8) (a) Huffman, M. A.; Fryszkowska, A.; Alvizo, O.; Borra-Garske, M.; Campos, K. R.; Canada, K. A.; Devine, P. N.; Duan, D.; Forstater, J. H.; Grosser, S. T.; Halsey, H. M.; Hughes, G. J.; Jo, J.; Joyce, L. A.; Kolev, J. N.; Liang, J.; Maloney, K. M.; Mann, B. F.; Marshall, N. M.; McLaughlin, M.; Moore, J. C.; Murphy, G. S.; Nawrat, C. C.; Nazor, J.; Novick, S.; Patel, N. R.; RodriguezGranillo, A.; Robaire, S. A.; Sherer, E. C.; Truppo, M. D.; Whittaker, A. M.; Verma, D.; Xiao, L.; Xu, Y.; Yang, H. Science 2019 366, 1255-1259. (b) Fernandez-Lucas, J. Appl. Microbiol Biotechnol 2015, 99, 4615-4627. (c) Birmingham, W. R.; Starbird, C. A.; Panosian, T. D.; Nannemann, D. P; Iverson, T. M.; Bachman, B. O. Nat. Chem. Bio. 2014, 10, 392-399

(9) (a) Ferro, A.J.; Barrett, A.; Shapiro, S.K. J. Biol Chem. 1978, 253, 6021-6025. (b) Albers, E. IUBMB Life 2009, 61, 1132-1142.

(10) Tozzi, M. G.; Camici, M.; Mascia, L.; Sgarrella, F.; Ipata, P. L. FEBS J. 2006, 273, 1089-1101.

(11) (a) Lipmann, F.; Tuttle, L. C. J. Biol. Chem. 1944, 153, 571-582. (b) Kazlauskas, R. J.; Whitesides, G. M. J. Org. Chem. 1985, 50, 1069-1076.

(12) Sedewitz, B.; Schleifer, K.H.; Gotz F. J. Bacteriol. 1984, 160, 273 278 .

(13) Kim, D.M.; Swartz, J.R. Biotechnol. Bioeng. 1999, 66, 180-188.

(14) Rose, I. A.; Grunberg-Manago, M.; Korey, S. R.; Ochoa, S. J. J. Biol Chem. 1953, 211, 737-756.
(15) Nomura, M.; Sato, T.; Washinosu, M.; Tanaka, M.; Asao, T.; Shuto, S.; Matsuda, A. Tetrahedron, 2002, 58, 1279-1288.

(16) Birkofer, L.; Ritter, A., Angew. Chem., Int. Ed. 1965, 4, 417. (b) Fleming, I., Chem. Soc. Rev. 1981, 10, 83. (c) Lalonde, M.; Chan, T. H., Synthesis 1985, 817 